\title{
Impact of Phase Noise and Compensation Techniques in Coherent Optical Systems
}

\author{
Giulio Colavolpe, Senior Member, IEEE, Tommaso Foggi, Enrico Forestieri, Member, IEEE, and Marco Secondini
}

\begin{abstract}
One of the most severe impairments that affect coherent optical systems employing high-order modulation formats is phase noise due to transmit and receive lasers. This is especially detrimental in uncompensated links, where an ideal compensator for channel distortions and laser phase noise should first eliminate receive phase noise, then equalize channel distortions, and only later compensate for transmit phase noise. Unfortunately, the simultaneous presence of transmit and receive phase noise makes very difficult to discriminate between them, even in the presence of a pilot tone. Moreover, the picture is different for optical systems using single-carrier or orthogonal frequency division multiplexing, where transmit and receive phase noise components may have a different impact. All these aspects are analyzed and discussed in this paper. A novel digital coherence enhancement (DCE) technique, able to significantly reduce the phase noise of transmit or receive lasers by using an interferometric device plus a very simple electronic processing, is also described. The performance of this technique and the statistical properties of the residual phase noise are analytically derived and verified by simulations, showing a high increase of the maximum bit-rate-distance product. The practical implementation of DCE is finally discussed and some alternative implementation schemes are presented.
\end{abstract}

Index Terms-Coherent detection, group velocity dispersion (GVD), optical communication, orthogonal frequency division multiplexing (OFDM), phase noise.

\section{INTRODUCTION}

$\mathbf{I}$ $\mathrm{N}$ recent years, coherent optical systems have come back into interest for the upgrade of transmission links towards $100 \mathrm{~Gb} / \mathrm{s}$ [2]. Through a coherent front end, high-order phase and phase-amplitude modulation formats can be exploited to carry more information per symbol period, and advanced signal processing can be also envisaged at the receive end, thus enabling the transmission of extremely-high bit rates [3], [4].

As the target of $100 \mathrm{~Gb} / \mathrm{s}$ seems to be reached and demonstrated, research activity is now focusing on the next advancement, $400 \mathrm{~Gb} / \mathrm{s}$ or even $1 \mathrm{~Tb} / \mathrm{s}$. In the light of such an upgrade, other transmission techniques, alternative to standard singlecarrier (SC) schemes, have been proposed, since they seemingly allow to more easily scale to higher bit rates, such as orthogonal frequency division multiplexing (OFDM) [5], whose

Manuscript received April 08, 2011; revised June 28, 2011; accepted July 30, 2011. Date of publication August 12, 2011; date of current version September 02,2011 . This work was supported by Ericsson AB and has been presented in part at the IEEE Global Communications Conference 2010 [1].

G. Colavolpe is with University of Parma, Dipartimento di Ingegneria dell'Informazione, I-43100 Parma, Italy.

T. Foggi is with CNIT Research Unit, I-43100 Parma, Italy.

E. Forestieri and M. Secondini are with Scuola Superiore Sant'Anna, I-56124

Pisa, Italy (e-mail: marco.secondini@sssup.it).

Color versions of one or more of the figures in this paper are available online at http://ieeexplore.ieee.org.

Digital Object Identifier 10.1109/JLT.2011.2164237 success and popularity in wireless communications several authors push to repeat in optical communications [6]-[8]. Besides more general considerations about the debate between SC and OFDM (see [9]), one of the most severe impairment that affects coherent systems employing high-order modulation formats is the presence of phase noise (PN) [10], [11], introduced by both transmit and receive lasers - the former also present in widespread intensity-modulation direct-detection systems, the latter peculiar to coherent systems.

Lasers PN, commonly modeled as a Wiener process [12], is due to the instabilities of optical light sources, whose linewidth can vary from a few hundreds $\mathrm{kHz}$ to a few $\mathrm{MHz}$, and can prevent correct detection of transmitted data. Several works have addressed the sensitivity to PN of SC coherent systems with inline dispersion compensation and discussed some countermeasures to reduce its detrimental effects [4], [13]-[15] (see also [16]-[18] and references therein for the effects and countermeasures on wireless systems). In OFDM systems, the effect of PN is even more severe [9] and represents a major comparison aspect between OFDM and SC.

Nowadays, the effect of PN is increased by the trend to design uncompensated long-haul optical links, as these links would benefit from milder nonlinear effects [19]. As an example, considering an SC transmission scheme in the presence of a highly dispersive channel, the complex digital equalizer that compensates for the group velocity dispersion (GVD) of thousands kilometers of fiber reverses the effect that the dispersive channel has on transmit PN, whereas receive PN, which only sees the equalizer, induces a detrimental residual intersymbol interference (ISI) (see [20] and references therein). Similar considerations hold for OFDM, as discussed later. As we will show, the transmission, along with the information-bearing signal, of an unmodulated carrier (pilot tone) used to estimate PN, does not solve the problem but simply move it from receive to transmit PN.

In this paper, we discuss all these aspects and extend the analysis of the digital coherence enhancement (DCE) technique, recently proposed in [1], able to significantly reduce transmit or receive PN by using an interferometric device plus very simple electronic processing. The statistical properties of the residual PN after DCE are derived and used to obtain a simple criterion for the estimation and optimization of the achievable performance. Practical aspects are also discussed and some alternative implementation schemes are presented. Finally, the DCE effectiveness for SC and OFDM systems on different links is tested and compared to that of other existing techniques through numerical simulations, showing that DCE is able to remarkably loosen the constraints on the adopted lasers in dispersion-uncompensated systems. The paper is organized as follows. After the description of the adopted system model in Section II, 


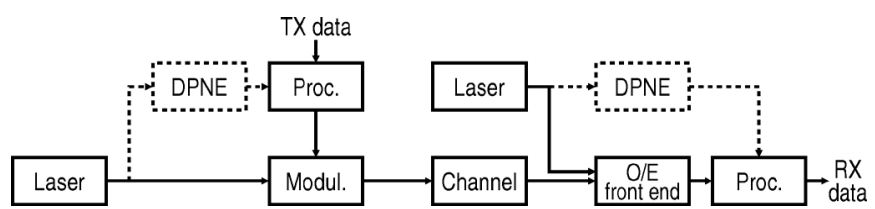

Fig. 1. System scheme.

Section III summarizes and discusses the techniques, available in the literature, considered in this paper for combating PN. Section IV introduces and analyzes the DCE technique, whose practical implementation in SC and OFDM systems is discussed in Section V. Simulation results are shown in Section VI and, finally, conclusions are drawn in Section VII.

\section{System Model}

We consider long-haul optical transmissions using polarization multiplexing, where two independent data streams, properly differentially encoded when necessary, either undergo direct linear modulation, in an SC transmission scheme, or are first processed by an inverse fast Fourier transform (IFFT) algorithm and then, after the insertion of the cyclic prefix (CP), are properly modulated onto the optical carrier, in an OFDM system. A schematic block diagram of the system is reported in Fig. 1. The optional differential phase noise estimation (DPNE) blocks are required for the implementation of the DCE technique and will be introduced in Section IV. As in [9], in the numerical results we will consider classical differentially encoded quadrature phase-shift keying (QPSK) signals. The modulated signal is then launched on two orthogonal states of polarization (SOPs) of a single mode fiber (SMF). We will denote by $\mathbf{x}_{\ell}=\left[x_{1, \ell}, x_{2, \ell}\right]^{T}$ the vector collecting the transmitted samples on these two orthogonal SOPs at symbol time $\ell .{ }^{1}$ These samples are the differentially encoded symbols in the SC case, or the samples after IFFT and CP insertion in the OFDM case.

We consider the receiver as composed of an analog opto-electronic $(\mathrm{O} / \mathrm{E})$ front end, devoted to signal demodulation and conversion from the optical to the electrical domain, and a digital part devoted to electronic processing. After a preliminary optical filtering, two orthogonal SOPs are split through a polarization beam slitter (PBS). They are then separately combined with the optical field of a local oscillator (LO) laser in a $2 \times 490^{\circ}$ hybrid [21] and detected with two balanced photodetectors. In this way, the two received signals, one for each SOP, are converted in the electrical domain, in practice performing a frequency conversion. We will collect the two received signals $r_{1}(t)$ and $r_{2}(t)$ in the electrical domain in a vector $\mathbf{r}(t)=\left[r_{1}(t), r_{2}(t)\right]^{T}$. The optical channel can be described as a multiple-input multiple-output (MIMO) channel which, in the linear regime, i.e., in the presence of GVD and polarization mode dispersion (PMD) and absence of nonlinear propagation effects, is described in the frequency domain by a $2 \times 2$ Jones matrix. We will denote by

\footnotetext{
${ }^{1}$ In the following $(\cdot)^{T}$ denote transpose, $\mathbf{I}$ the $2 \times 2$ identity matrix, and $\otimes$ denotes convolution.
}

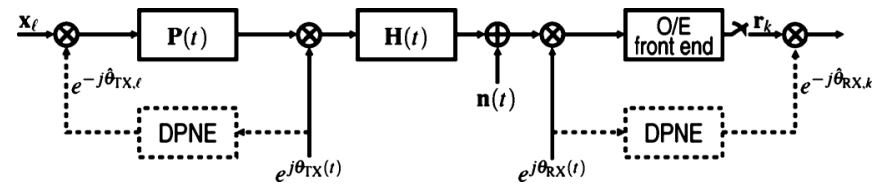

Fig. 2. Low-pass equivalent model of the system.

$\mathbf{H}(t)$ the inverse Fourier transform of this Jones matrix. According to the low-pass equivalent model of Fig. 2 , in the absence of the optional DPNE blocks, the received vector is

$$
\mathbf{r}(t)=\left\{\mathbf{H}(t) \otimes\left[e^{j \theta_{\mathrm{TX}}(t)} \sum_{\ell} \mathbf{P}(t-\ell T) \mathbf{x}_{\ell}\right]\right\} e^{j \theta_{\mathrm{RX}}(t)}
$$

where $T$ is the symbol interval, $\mathbf{P}(t)=p(t) \mathbf{I}, p(t)$ being the transmitted pulse, $\theta_{\mathrm{TX}}(t)$ and $\theta_{\mathrm{RX}}(t)$ are the transmit and receive $\mathrm{PN}$, respectively, and $\mathbf{n}(t)=\left[n_{1}(t), n_{2}(t)\right]^{T}$ is a vector collecting two independent complex noise components, representing the lowpass equivalent of the filtered amplified spontaneous emission (ASE) noise components, assumed dominant over thermal and shot noise, on two orthogonal SOPs. Transmit and receive PN stochastic processes are modeled according to the Wiener model with full-width half-maximum (FWHM) linewidths $\nu_{\mathrm{TX}}$ and $\nu_{\mathrm{RX}}$, respectively. Since in this paper we will focus on the effect of $\mathrm{PN}$, in (1) we implicitly assumed that a perfect frequency estimation and compensation has been performed, for instance through the use of the automatic frequency control loop described in [4].

A discrete-time sufficient statistics is then obtained through oversampling in the SC case [4], whereas one sample per symbol interval is sufficient in the OFDM scheme since, in this case, oversampling is accounted for through the use of virtual subcarriers. Denoting by $T_{c}$ the sampling interval $\left(T_{c}=T\right.$ in OFDM), we define $\mathbf{r}_{k}=\mathbf{r}\left(k T_{c}\right)$. In the considered SC system, dispersion compensation is performed by a two-dimensional fractionally-spaced linear equalizer which, if properly designed, can perfectly remove the ISI due to GVD and PMD [4]. This two-dimensional equalizer can be suitably implemented in the frequency domain for complexity-saving purposes [9]. The adoption of the asynchronous strategies for detection and for the adjustment of the equalizer taps described in [4] allows to remarkably increase the robustness to PN. Instead, in the OFDM scheme, blocks of signal samples undergo FFT, are then processed with a one-tap equalizer and, finally, detection of the transmitted sequences takes place. Details on one possible OFDM receiver architecture with the relevant algorithms can be found in [9].

\section{PN ESTIMATION AND COMPENSATION}

Possible countermeasures to mitigate PN effects and the relative robustness of SC and OFDM systems have been deeply investigated in the wireless and optical literature of the last decades (see [16]-[18], [22] and references therein). We will 
consider here the following PN compensation techniques which are relevant in high-speed optical links.

For OFDM systems, we will consider the pilot-tone-based compensation method described in [23]. Some data subcarriers are sacrificed to transmit an unmodulated pilot tone (PT) that, properly filtered, provides the required reference for phase estimation and compensation before FFT at the receiver. In this case, the power allocated to the unmodulated subcarrier must be optimized. In fact, the higher its power, the more reliable the carrier estimate, but also the higher the energy not associated to data (and hence wasted)

The PT-based compensation could, in principle, be employed also in SC schemes. Indeed, through proper line coding, the power spectral density (PSD) of the transmitted signal can be shaped to have a null where an unmodulated carrier can be placed. As an example, adopting duobinary encoding (properly extended to complex symbol constellations) the lowpass equivalent of the transmitted signal will have a null at the frequency $1 / 2 T$. This kind of line coding will imply a performance loss of a few tenth of $\mathrm{dB}$ if a proper Viterbi decoder, with 4 states in the case of QPSK, is employed [24]. As we will see, this technique is either not necessary or useless. On the contrary, a technique very useful to increase the receiver robustness is the asynchronous detection algorithm described in [4].

\section{A. Dispersion-Compensated Links}

In the presence of a very limited chromatic dispersion, as in links with inline dispersion compensation, the memory length $T_{H}$ of the channel $\mathbf{H}(t)$ in Fig. 2 is short compared to the time scales on which phase variations due to receive PN take place, i.e., $T_{H} \ll 1 / \nu_{\mathrm{RX}}$. Therefore, the operations of phase noise multiplication and channel convolution in (1) commute and the received signal can be approximated as

$$
\mathbf{r}(t) \simeq \mathbf{H}(t) \otimes\left\{e^{j\left[\theta_{\mathrm{TX}}(t)+\theta_{\mathrm{RX}}(t)\right]} \sum_{\ell} \mathbf{P}(t-\ell T) \mathbf{x}_{\ell}\right\}+\mathbf{n}(t)
$$

meaning that things go as if the transmitted signal is first corrupted by an equivalent $\mathrm{PN}$, given by the sum of transmit and receive $\mathrm{PN}$, and then is convolved with the channel matrix $\mathbf{H}(t)$ which, in the presence of GVD and PMD, is unitary. In this case, thanks to the mentioned asynchronous strategies for detection and for the adjustment of the equalizer taps described in [4], the described SC scheme can achieve perfect channel equalization and exhibits a very good robustness to PN. The impact of PN depends on the modulation format, signal-to-noise ratio, and on the product $\left(\nu_{\mathrm{TX}}+\nu_{\mathrm{RX}}\right) T$, and is therefore inversely proportional to the symbol rate $R_{s}=1 / T$. Provided that short OFDM symbols are employed, also the OFDM system exhibits a limited performance degradation for laser linewidths of practical interest [9]. When larger OFDM symbols are employed, a possible solution to improve the robustness against $\mathrm{PN}$ is the use of the described PT-based compensation method.

\section{B. Dispersion-Uncompensated Links}

Neglecting polarization effects not relevant to the present discussion, in optical systems without inline compensation the channel transfer matrix may be written as

$$
\mathcal{F}\{\mathbf{H}(t)\}=\exp \left(-j 2 \pi^{2} \beta_{2} L f^{2}\right) \mathbf{I}
$$

being $\beta_{2}$ the dispersion parameter and $L$ the fiber length. In this case, the channel memory, approximately equal to the differential group delay at the extremes of the Nyquist band ${ }^{2}$

$$
T_{H} \simeq 2 \pi\left|\beta_{2}\right| L R_{s}
$$

increases with the bit-rate-distance product and can be very long (up to hundreds of symbols) for systems of practical interest. In particular, if the condition $T_{H} \ll 1 / \nu_{\mathrm{RX}}$ does not hold, phase noise multiplication and channel matrix convolution in (1) do not commute and (2) does not hold. Therefore, in SC systems, $\theta_{\mathrm{RX}}(t)$ should be separately compensated before channel equalization. Otherwise, after channel equalization, a residual ISI induced by the fluctuations of the signal phase over channel memory $T_{H}$ due to receive PN is present [26], [27]. In order to find a simple measure of this effect, we define the average variance of the fluctuations of the signal phase inside a time interval $T_{H}$ around a generic time $t$ with respect to its center value

$$
\overline{\sigma_{\theta}^{2}}=\frac{1}{T_{H}} \int_{-T_{H} / 2}^{T_{H} / 2} E\left\{[\theta(t+\xi)-\theta(t)]^{2}\right\} d \xi
$$

and assume that the signal-to-noise ratio (SNR) penalty is simply and monotonically related to it. Indeed, the analysis reported in the Appendix and numerical simulations in Section VI show that the SNR penalty is well approximated by

$$
\Delta \rho_{\mathrm{dB}}=10 \log _{10}\left(\frac{\rho}{\rho_{0}}\right) \simeq-10 \log _{10}\left(1-\rho_{0} \overline{\sigma_{\theta}^{2}}\right)
$$

where $\rho$ and $\rho_{0}$ are the required SNRs for a given bit-error rate (BER) in the presence and absence of phase noise, respectively. After a few calculations, reported in the Appendix, the value of (5) in the absence of any countermeasure can be simply related to the laser linewidth, residual dispersion and symbol rate through

$$
\overline{\sigma_{\theta}^{2}}=\pi \nu_{\mathrm{RX}} T_{H} / 2 \simeq \pi^{2}\left|\beta_{2}\right| L \nu_{\mathrm{RX}} R_{s} .
$$

In contrast to the case of compensated links, the impact of receive $\mathrm{PN}$ is directly proportional to the symbol rate. It is worth noting that (7) equals the variance of the equalization-enhanced phase noise defined in [26] for a QPSK system whose pulses are rectangularly shaped in frequency with bandwidth $1 /(2 T)$. As regards transmit $\mathrm{PN}$, its presence is not directly responsible for a significant performance degradation in dispersion-uncompensated SC systems. Indeed, if we were able to estimate and compensate receive $\mathrm{PN}$ before channel equalization by means of some non-data-aided method-e.g., the described PT-based

\footnotetext{
${ }^{2}$ As explained in [25], a dispersive fiber turns an input pulse $s_{\text {in }}(t)$ into the shape of its Fourier transform $S_{\text {in }}(f)$, such that $\left|s_{\text {out }}(t)\right| \propto$ $\left|S_{\text {in }}\left(t /\left(2 \pi \beta_{2} L\right)\right)\right|$. Hence, if $S_{\text {in }}(f) \simeq 0$ for $|f|>\kappa R_{s}$ the output pulse $s_{\text {out }}(t)$ approximately vanishes for $|t|>2 \pi\left|\beta_{2}\right| L \kappa R_{s}$.
} 
compensation - the equalizer would reverse the ISI due to the combined effect of transmit PN and channel filtering and the asynchronous detection algorithm would complete the work, compensating transmit PN after equalization, with a final impact comparable to the case of compensated links (proportional to $\nu_{\mathrm{TX}} / R_{s}$ and negligible for systems of practical interest). Unfortunately, it is the presence of transmit PN that makes the PT solution unfeasible. In fact, let us suppose that we transmit, along with the useful signal, a PT. The received signal $\mathbf{r}(t)$ becomes

$$
\begin{aligned}
\mathbf{r}(t)= & \gamma\left\{\mathbf{H}(t) \otimes\left[e^{j \theta_{\mathrm{TX}}(t)} \sum_{\ell} \mathbf{P}(t-\ell T) \mathbf{x}_{\ell}\right]\right\} e^{j \theta_{\mathrm{RX}}(t)} \\
& +\sqrt{1-\gamma^{2}}\left[\mathbf{H}(t) \otimes e^{j\left[2 \pi f_{p} t+\theta_{\mathrm{TX}}(t)\right]} \mathbf{p}\right] e^{j \theta_{\mathrm{RX}}(t)} \\
& +\mathbf{n}(t)
\end{aligned}
$$

where parameter $\gamma$ takes into account the power allocation between the useful signal and the unmodulated carrier, frequency $f_{p}$ the position of the PT, and unit vector $\mathbf{p}$ its SOP. Considering the fiber transfer matrix in (3), we note that, while the useful signal (first term), with a bandwidth of about $R_{s}$, is strongly affected by GVD and sees a channel with memory $T_{H}$, the PT (second term), due to its much narrower bandwidth $\nu_{\mathrm{TX}}$, is not affected by GVD (i.e., $\beta_{2} L \pi^{2} \nu_{\mathrm{TX}}^{2} / 2 \ll 1$ ) and its propagation can be accurately approximated by taking a first-order Taylor expansion of (3) around frequency $f=f_{p}$, obtaining

$$
\begin{aligned}
\mathbf{r}(t) \simeq & \gamma\left\{\mathbf{H}(t) \otimes\left[e^{j \theta_{\mathrm{TX}}(t)} \sum_{\ell} \mathbf{P}(t-\ell T) \mathbf{x}_{\ell}\right]\right\} e^{j \theta_{\mathrm{RX}}(t)} \\
& +\sqrt{1-\gamma^{2}} e^{j\left[2 \pi f_{p} t+\theta_{\mathrm{TX}}\left(t-t_{p}\right)+\theta_{\mathrm{RX}}(t)-\phi_{p}\right]} \mathbf{p}+\mathbf{n}(t)
\end{aligned}
$$

where $t_{p}=2 \pi \beta_{2} L f_{P}$ and $\phi_{p}=2 \pi^{2} \beta_{2} L f_{p}^{2}$. In practice, things go as if the PT propagates through a memoryless channel with group delay $t_{p}$ and phase shift $\phi_{p}$, while the useful signal propagates through a dispersive channel with memory $T_{H}$. This fact has two significant consequences. Firstly, since there is no way to distinguish between transmit and receive PN by observing the PT, $\theta_{\mathrm{RX}}(t)$ cannot be separately estimated and compensated before equalization. Secondly, since at time $t$ the PT is affected by $\theta_{\mathrm{RX}}(t)$ and $\theta_{\mathrm{TX}}\left(t-t_{p}\right)$ while the useful signal is affected by $\theta_{\mathrm{RX}}(t)$ and by the values that transmit PN assumes in a time interval $T_{H}$ around $t$, the higher the channel dispersion, the lower the correlation between the phase of the PT and the PN affecting the useful signal. Therefore, if we use the phase extracted from the PT to compensate for PN before equalization, we do not get any benefit. In fact, neglecting the irrelevant constant phase shift $\phi_{p}$ and approximating the phase extracted by an (ideal) PN estimation algorithm working on the PT as $\theta_{\mathrm{RX}}(t)+\theta_{\mathrm{TX}}\left(t-t_{p}\right)$, the first term perfectly compensates for receive PN, while the second term, being practically uncorrelated with the PN affecting the information-bearing component of the received signal, introduces an additional PN and causes ISI after equalization. In this case, the average variance of residual phase noise over channel memory is still given by (7), with $\nu_{\mathrm{Tx}}$ replacing $\nu_{\mathrm{RX}}$. Therefore, when transmit and receive lasers have the same linewidth, the impact of the new PN term $-\theta_{\mathrm{TX}}\left(t-t_{p}\right)$ introduced by the PT compensation method is as detrimental as the compensated PN term $\theta_{\mathrm{RX}}(t)$, making the PT method useless.

The picture is even worse in OFDM systems, which are strongly affected by both transmit and receive PN, whose variations over a time equal to the FFT block length - that should be significantly higher than $T_{H}$ to keep the spectral and energy loss due to CP negligible [9] — cause the onset of ICI after the FFT block at the receiver. Also in this case, the PT compensation method does not bring any benefit since, as demonstrated, it is not able to distinguish between transmit and receive PN. In addition, even if we were able to distinguish them and remove receive PN before the FFT block, performance would be still affected by transmit PN. Such a different behavior of SC and OFDM systems with respect to transmit PN will also reflect on the implementation of the PN compensation technique described in the next section.

\section{Digital Coherence Enhancement: OPERATION PRINCIPLE}

In this section, we describe the digital coherence enhancement (DCE) technique for the compensation of receive PN. The application of DCE for transmit PN cancellation will be discussed in the next section. The basic idea is that of measuring the random phase fluctuations of the laser before it is combined with the received signal by means of the differential phase noise estimator (DPNE) block reported in Fig. 3. The DPNE exploits an optical hybrid plus a simple electronic processing to measure the phase variations of the $\mathrm{LO}$ on a given time $\tau$ and, in this respect, can be regarded as a differential detector applied to the LO. The measured variations are then digitally removed from the received samples $\mathbf{r}_{k}$, such that the receiver works as if a LO with a higher coherence were used. Neglecting the amplitude noise, the optical carrier generated by the LO laser is

$$
e_{\mathrm{LO}}(t)=\sqrt{P_{\mathrm{LO}}} e^{j\left[2 \pi f_{\mathrm{LO}} t+\theta_{\mathrm{RX}}(t)\right]}
$$

where $P_{\mathrm{LO}}$ is its power, $f_{\mathrm{LO}}$ its frequency, and $\theta_{\mathrm{RX}}(t)$ the phase noise. The optical carrier is split by a beam splitter in two components. The first one, with power $(1-\alpha) P_{\mathrm{LO}}$, is combined with the received optical signal in the opto-electronic front end to get the electrical signal $\mathbf{r}(t)$. The second one, with power $\alpha P_{\mathrm{LO}}$, is sent to a sort of differential detector, made of a beam splitter, an optical delay $\tau$, a $90^{\circ}$ optical hybrid, and two balanced photodetectors, measuring its phase variations over a time $\tau$. As shown in the Appendix, the normalized complex output signal $y(t) \triangleq\left[x_{r}(t)+j x_{i}(t)\right] /\left(R \alpha P_{\mathrm{LO}} / 2\right)$ can be written as

$$
y(t)=\exp \left\{j\left[\theta_{\mathrm{RX}}(t)-\theta_{\mathrm{RX}}(t-\tau)+\phi\right]\right\}+w(t)
$$

where $\phi=2 \pi f_{\mathrm{LO}} \tau-\phi^{\prime}$ is an unknown slowly-varying phase shift that depends on the local oscillator frequency and on the phase shift $\phi^{\prime}$ between the delayed and undelayed optical carrier 
at the input of the optical hybrid, and $w(t)$ is a circularly symmetric zero-mean white (over the detector bandwidth) Gaussian noise with $\mathrm{PSD}^{3}$

$$
N_{w}=\frac{16 k_{B} \Theta F_{n}}{R_{L} R^{2}\left(\alpha P_{\mathrm{LO}}\right)^{2}}=\frac{4 N_{\mathrm{EP}}^{2}}{\left(\alpha P_{\mathrm{LO}}\right)^{2}}
$$

where $k_{B}$ is the Boltzmann constant, $\Theta$ the temperature, $R_{L}$ the load resistance of the photodetectors, $R$ its responsivity, $F_{n}$ the noise figure of an optional electrical amplifier, and $N_{\mathrm{EP}}$ the resulting noise equivalent power of the photodetectors, whose typical values are in the range of $1 \div 10 \mathrm{pW} / \sqrt{\mathrm{Hz}}$ [28]. Given $y(t)$ in (11) and an arbitrary initial time $t_{0}$, an unbiased estimate of the laser phase noise $\theta_{\mathrm{RX}}(t)$ can be obtained as

$$
\hat{\theta}(t)=\frac{1}{\tau} \int_{t_{0}}^{t+\tau / 2}[\angle y(x)-\hat{\phi}] d x
$$

where $\hat{\phi}$ is an unbiased estimate of $\phi$ obtained from a sufficiently long time-average of $\angle y(t)$. Indeed, as shown in the Appendix, the estimate in (13) can be rewritten as

$$
\hat{\theta}(t)=\theta_{\mathrm{RX}}(t)+\theta_{0}+\theta_{w}(t)+\theta_{d}(t)
$$

where $\theta_{0}$ is a constant phase error, therefore irrelevant, equal to the average phase in the initial time interval $\left[t_{0}-\tau, t_{0}\right] ; \theta_{w}(t)$ is a Wiener process, statistically equivalent to the phase noise that would be generated by a LO with linewidth $\nu_{\mathrm{eq}}=N_{w} /\left(4 \pi \tau^{2}\right)$, due to the cumulated photodetection noise; $\theta_{d}(t)$ is a stationary zero-mean Gaussian process with variance $\sigma_{d}^{2}=\pi \nu_{\mathrm{RX}} \tau / 6$, and is the phase error due to measuring the average phase in the interval $[t-\tau / 2, t+\tau / 2]$ in place of the instantaneous phase $\theta_{\mathrm{RX}}(t)$. By using the estimate in (13) to remove the phase noise of the local oscillator from the detected signal in (1), we eventually get a signal $\mathbf{r}^{\prime}(t)=\mathbf{r}(t) \exp [-j \hat{\theta}(t)]$ that - apart from a constant phase rotation $\theta_{0}$ that is irrelevant both in SC systems, due to the presence of the asynchronous detection strategy, and in OFDM systems, due to the channel estimator-is corrupted by a residual phase noise $\theta_{e}(t)=\theta_{d}(t)+\theta_{w}(t)$. In particular, changing the delay $\tau$ has an opposite effect on $\theta_{d}(t)$ and $\theta_{w}(t)$ - the former increases with $\tau$, since phase variations on time scales shorter than $\tau$ are not compensated by DCE; the latter decreases with $\tau$, since photodetection noise is accumulated faster and on a larger bandwidth. Its optimal choice is a trade off to minimize the total residual phase noise $\theta_{e}(t)$ after DCE. The impact of $\theta_{e}(t)$ on system performance depends on several factors, such as the adopted modulation format and detection strategy. In general, slow (with respect to the equalizer memory or OFDM symbol) phase fluctuations of the received signal $\mathbf{r}(t)$ pass unchanged through the equalizer or FFT operation and do not affect the performance when a proper asynchronous detection strategy or channel estimator are present. On the other hand, fast fluctuations cause ISI and ICI in SC and OFDM systems, respectively. Therefore, in order to derive a simple but general rule to estimate the performance of the DCE technique and optimize the delay $\tau$, we assume that system performance can be still characterized by the average variance of

\footnotetext{
${ }^{3}$ Optical components inside the DPNE may introduce additional insertion losses that are accounted for by decreasing the value of $\alpha$.
}

the fluctuations of the signal phase over channel memory $T_{H}$ defined in (5). After some calculations, reported in the Appendix, we obtain

$$
\overline{\sigma_{\theta \mathrm{DCE}}^{2}} \simeq \frac{\pi \nu_{\mathrm{RX}} \tau}{3}+\frac{N_{w} T_{H}}{8 \tau^{2}} .
$$

The first term of (15) accounts for $\theta_{d}(t)$, i.e., for the error generated by phase variations that are faster than $\tau$, and is proportional to laser linewidth $\nu_{\mathrm{RX}}$ and measuring time $\tau$. The second term accounts for $\theta_{w}(t)$, that is the error generated by the cumulated photodetection noise in the interval $T_{H}$, and is proportional to the number of "independent measures" during time $T_{H}$, i.e., the ratio $T_{H} / \tau$, and inversely proportional to the signal-to-noise ratio of the signal $y(t)$ in (11) on a bandwidth $1 / \tau$, i.e., the ratio $\tau / N_{w}$. From (15), the optimum delay is

$$
\tau_{\mathrm{opt}}=\sqrt[3]{\frac{3 N_{w} T_{H}}{4 \pi \nu_{\mathrm{RX}}}}
$$

with a minimum average variance

$$
{\overline{\sigma_{\text {DCE }, \text { min }}^{2}}} \simeq 0.45 \sqrt[3]{N_{w}\left(\pi \nu_{\mathrm{RX}}\right)^{2} T_{H}} .
$$

Fig. 4 reports the required receive-laser linewidth $\nu_{\mathrm{RX}}$ as a function of channel memory $T_{H}$ in the absence of DCE-obtained from (7) - or in the presence of DCE—obtained from (17) for different values of electronics noise PSD $N_{w}$-to have $\overline{\sigma_{\theta}^{2}}<$ 0.017 , which, as obtained from (6) and confirmed by numerical simulations in Section VI, guarantees a penalty due to receive PN lower than $0.5 \mathrm{~dB}$ at BER $=10^{-3}$ for both $\mathrm{SC}$ and OFDM systems deploying a QPSK modulation format. For a typical photodetector $N_{\mathrm{EP}}$ value of $10 \mathrm{pW} / \sqrt{\mathrm{Hz}}$, the reported values of $N_{w}$ correspond to an available optical power $\alpha P_{\mathrm{LO}}$ at the input of the DPNE of $-12,-17$, and $-22 \mathrm{dBm}$, respectively. For DCE curves, the corresponding value of $1 / \tau_{\mathrm{opt}}$ which, as shown in the Appendix, is also an indication of the required bandwidth and sampling rate for the DPNE block, is reported on the right axis. For each value of $T_{H}$, the upper axis below the graph shows the corresponding distance-bit-rate product $L R_{b}=2 L R_{s}$ on a standard single-mode fiber $\left(\beta_{2} \simeq\right.$ $22 \mathrm{ps}^{2} / \mathrm{km}$ ) for an SC system deploying polarization multiplexing and DQPSK format. It is worth reminding that, while in SC systems channel memory $T_{H}$ is set by chromatic dispersion according to (4), in OFDM systems it is approximately equal to the FFT block length, that should be significantly higher than $T_{H}$ to keep the spectral and energy loss due to CP negligible. For instance, in the numerical simulations shown in Section VI, the FFT block length is taken approximately 20 times longer than $T_{H}$ to keep spectral and energy loss below $5 \%$. Therefore, the corresponding bit-rate-distance product should be divided by 20 with respect to $\mathrm{SC}$, as reported in the lower axis. Without DCE, common DFB lasers with a-few-MHz linewidth can be deployed only for moderate amounts of channel memory, e.g., SC systems up to $R_{b}=100 \mathrm{~Gb} / \mathrm{s}, L=1000 \mathrm{~km}$ or OFDM systems up to $R_{b}=100 \mathrm{~Gb} / \mathrm{s}, L=50 \mathrm{~km}$. Since the required linewidth from (7) scales linearly with the inverse of $T_{H}$, external cavity lasers with a-few-hundreds-kHz linewidth can be adopted to increase the bit-rate-distance product up to one order of magnitude, while higher products would require even better lasers. On the other hand, with DCE, the required linewidth from 


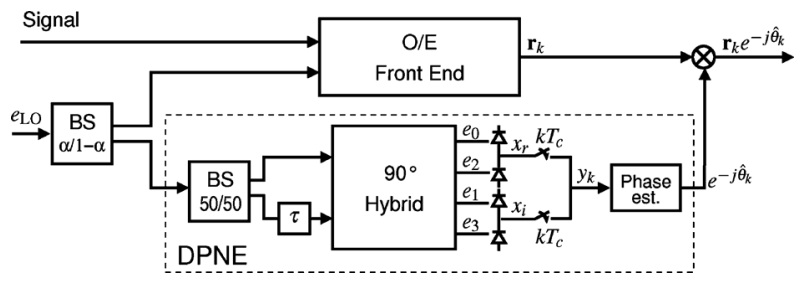

Fig. 3. Scheme for the implementation of receive DCE.

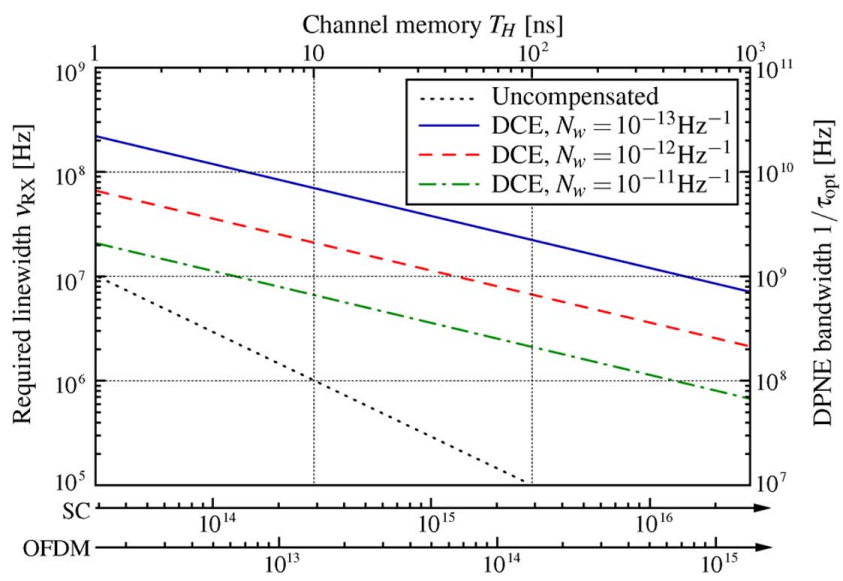

Bit-rate-distance product for DP-QPSK on standard fiber $[(\mathrm{b} / \mathrm{s}) \cdot \mathrm{km}]$

Fig. 4. Required laser linewidth $\nu_{\mathrm{Rx}}$ (left axis) and corresponding DPNE bandwidth $1 / \tau_{\text {opt }}$ (right axis, only for DCE) as a function of channel memory $T_{H}$ (and corresponding bit-rate-distance product on the lower axes) to have an average PN variance $\sigma_{\theta}^{2}=0.017$ (i.e., $\sim 0.5 \mathrm{~dB}$ penalty for a QPSK system at $\mathrm{BER}=10^{-3}$ ) without DCE (uncompensated) or with DCE and different values of the normalized noise $\operatorname{PSD} N_{w}$.

(17) is much higher and scales only with the square root of the inverse of $T_{H}$. Therefore, typical DFB lasers can be deployed even for an extremely long channel memory (e.g., OFDM with $R_{b}>1 \mathrm{~Tb} / \mathrm{s}, L>1000 \mathrm{~km}$ ), practically solving the problem of PN for any uncompensated system. We also note that, with DFB lasers, the required DPNE bandwidth is below $1 \mathrm{GHz}$, i.e., much narrower than receiver bandwidth, making the expected cost of the DPNE optoelectronic components small with respect to receiver. Although a much wider linewidth could be tolerated in principle, in this case the effect of transmit PN (not included in Fig. 4) would become significant and, in addition, the bandwidth and cost of DPNE would increase.

\section{Digital COHEREnce Enhancement: PRACTICAL IMPLEMENTATION}

In this Section we discuss the implementation of DCE in SC and OFDM systems, addressing the problem of both receive and transmit laser phase noise cancellation and considering some simplified schemes for the DPNE. Finally, we propose a different phase noise compensation strategy, based on the insertion of a couple of PTs, and show that, on dispersion-uncompensated links, this technique is practically equivalent to the DCE technique described in the previous section.

\section{A. DPNE Implementation}

The implementation of the DPNE block reported in Fig. 3, whose experimental demonstration we have recently reported in [29], makes use of a BS, an optical delay, a $90^{\circ}$ optical hybrid, and a couple of balanced photodetectors to measure the LO phase variations over a given time $\tau$. Some simplified implementations are also possible. For instance, the couple of balanced photodetectors can be replaced by two single photodetectors. In this case, after removing an irrelevant constant term, the normalized complex signal $y(t)$ is still given by (11) and all the results obtained in the previous section still hold, provided that the optical power in (12) is divided by two. An even simpler scheme is obtained by replacing the BS, the optical hybrid, and the couple of balanced photodetectors with an optical band-pass filter, slightly detuned with respect to the LO carrier frequency, and a photodiode. Assuming that the transfer function of the filter is approximately linear around the carrier frequency, $H(f) \simeq H\left(f_{\mathrm{LO}}\right)\left[1+\pi \beta\left(f-f_{\mathrm{LO}}\right)\right]$, the photodetected signal is

$$
y(t) \simeq c\left[1+\frac{\beta}{2} \frac{d \theta_{\mathrm{RX}}(t)}{d t}\right]^{2}+w(t)
$$

with $c=R \alpha P_{\mathrm{LO}}\left|H\left(f_{\mathrm{LO}}\right)\right|^{2}$. Equation (18) shows that the detected signal $y(t)$ depends quadratically on the time derivative of the LO phase $\theta_{\mathrm{RX}}$, which can be therefore still estimated by (13), with $\tau=\beta$ but replacing $\angle y(t)$ with $\sqrt{y(t) / c}$, where the constant $c$ is obtained from a long time average of $y(t)$. Also in this case, the choice of $\beta$ is the result of a trade off intended to minimize the estimate error. Indeed, cumulated photodetection noise generates a Wiener-like noise, that increases when decreasing $\beta$. At the same time, the linearity of $H(f)$ can be guaranteed only on a limited frequency range around $f_{\mathrm{LO}}$, which cannot exceed the interval $\left[f_{\mathrm{LO}}-1 /(\pi \beta), f_{\mathrm{LO}}+1 /(\pi \beta)\right]$, meaning that phase variations on time scales shorter than $\beta$ are not correctly estimated (are filtered out by the filter). Although the accuracy of the phase estimate provided by this scheme is, at least theoretically, similar to that obtained with the DPNE of Fig. 3, the actual performance depends significantly on the exact shape of the filter and is sensitive to deviations of the LO carrier frequency from $f_{\mathrm{LO}}$.

In a practical implementation of DPNE, the signal $y(t)$ in (11) or (18) is sampled (and quantized) before being processed, and the phase estimator in (13) is replaced by the digital estimator

$$
\hat{\theta}_{k}=\hat{\theta}_{k-1}+\frac{T_{c}}{\tau}\left(\angle y_{k}-\hat{\phi}_{k}\right)
$$

where $y_{k}=y\left(k T_{c}\right)$ and $\hat{\phi}_{k}$ is the average value of $\angle y_{k}$ over a large number of samples. The compensated samples are eventually obtained as $\mathbf{r}_{k}^{\prime}=\mathbf{r}_{k} \exp \left(-j \hat{\theta}_{k}\right)$. Provided that photodetector bandwidth and sampling rate in the DPNE are large enough to obtain a set of sufficient statistics of (11), and that quantization noise is negligible with respect to $w(t)$, the results obtained so far for the analog estimator in (13) are valid also for the digital one in (19). Indeed, as shown in the Appendix, the information bearing component of the signal $y(t)$ in (11) is practically confined within a bandwidth $1 / \tau$. Therefore, a set of sufficient statistics is obtained by using photodetectors with a bandwidth $B \geq 1 / \tau$ and sampling $y(t)$ at rate $2 B$, while phase estimates $\hat{\theta}_{k}$ at rate $1 / T_{c}$ can be obtained through interpolation. Hence, all the required DPNE components (photodetectors, 
ADCs, digital processors) can operate at a significantly lower speed than that required for the opto-electronic front-end and digital processing in the receiver, as indicated in Fig. 4.

In dispersion-uncompensated SC systems, the described DCE technique can be deployed at the receiver, as shown in Fig. 3, to estimate and remove receive $\mathrm{PN}$ before the equalizer, while transmit PN is handled by the asynchronous detection strategy after the equalizer. Instead, in OFDM systems, deploying the DCE technique at the receiver is not an effective solution since it does not solve the problem of transmit PN. In fact, according to (9), the phase extracted from the PT at the receiver can be directly used to compensate for receive PN but not for transmit PN. Therefore, two different solutions are available. The first one is deploying DCE at the transmitter to cancel transmit PN before propagation through the channel, while receive $\mathrm{PN}$ is estimated and compensated for at the receiver, before the FFT operation, by using the estimate from the PT. The second one is deploying DCE both at transmitter and receiver, as shown in Fig. 1, thus increasing the hardware complexity but avoiding the insertion of the PT. Transmit-end DCE is implemented by using the same DPNE block of Fig. 3 to measure phase variations of the transmit laser, and by applying the phase compensation term (19) to the digital samples $\mathbf{x}_{\ell}$, after IFFT and CP insertion, right before digital-to-analog conversion. Indeed, in OFDM systems, as opposed to SC systems, the digital processing capabilities and high-resolution digital-to-analog conversion required to implement the DCE technique at transmit end are already available.

Both in the receive or transmit implementation, either for SC or OFDM systems, phase noise cancellation requires synchronization between the received (or transmitted) signal $\mathbf{r}(t)$ and the phase estimate $\hat{\theta}(t)$, which, according to (13), is achieved when integration is performed up to $t+\tau / 2$. It is easy to show that a synchronization error $\Delta \tau$ would increase the average variance in (15) by $2 \pi \nu_{\mathrm{RX}} \Delta \tau$ and would be negligible provided that $\Delta \tau \ll \tau$. However, a fine tuning of the analog optical and electrical delays is not required, since the final delay between samples $\mathbf{r}_{k}$ and $\hat{\theta}_{k}$ can be easily measured and compensated (within one symbol time) by digitally delaying one of them.

\section{B. DCE Based on Two Pilot Tones}

The DCE implementation based on the DPNE block of Fig. 3 (or its simplified variants) requires some additional optoelectronic components. Here, an alternative solution is presented, based on the insertion of a couple of PTs, that can be used to implement the DCE technique without additional optoelectronic components. At transmit end, two PTs at frequencies $\pm f_{p}$ are inserted on the baseband signal. In OFDM systems, this is easily accomplished by sacrificing some subcarriers, while in SC systems - as for the single PT case - by creating spectral nulls at the desired frequencies in the PSD of the transmitted signal through proper line coding. ${ }^{4}$ According to the analysis of Section III.B, the received signal can be expressed as in (9), but for an additional PT at frequency $-f_{p}$, that propagates with same phase shift $\phi_{p}=2 \pi^{2} \beta_{2} L f_{p}^{2}$ and opposite group delay

\footnotetext{
${ }^{4}$ As an example, partial-response signaling [30], such as modified duobinary encoding, can be adopted to create spectral nulls at desired frequencies and can be decoded by a Viterbi decoder [31] with negligible performance loss and a small increase in complexity.
}

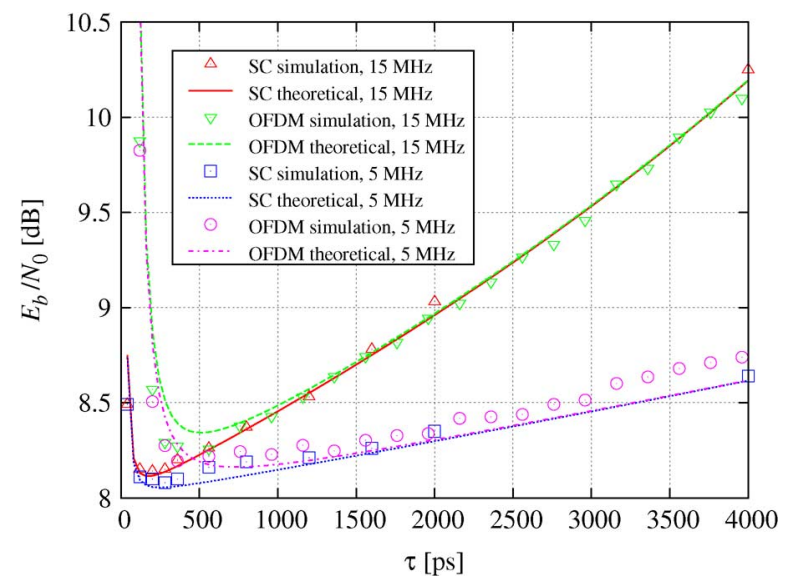

Fig. 5. Required $E_{b} / N_{0}$ versus DPNE delay $\tau$ to obtain a BER of $10^{-3}$ for $100 \mathrm{~Gb} / \mathrm{s} \mathrm{SC}$ or OFDM transmission and different laser linewidth $\nu_{\mathrm{RX}}$.

$-t_{p}=-2 \pi \beta_{2} L f_{p}$ with respect to the PT at frequency $f_{p}$. Therefore, the phases extracted from a couple of PLLs operating on the received signal and locked to the PTs at frequencies $\pm f_{p}$ can be approximated, respectively, as $\hat{\theta}_{ \pm}(t) \simeq \theta_{\mathrm{RX}}(t)+$ $\theta_{\mathrm{TX}}\left(t \mp t_{p}\right)$, such that

$$
\begin{aligned}
& y_{1}(t)=\hat{\theta}_{-}(t)-\hat{\theta}_{+}(t) \simeq \theta_{\mathrm{TX}}\left(t+t_{p}\right)-\theta_{\mathrm{TX}}\left(t-t_{p}\right) \\
& y_{2}(t)=\hat{\theta}_{+}(t)-\hat{\theta}_{-}\left(t-2 t_{p}\right) \simeq \theta_{\mathrm{RX}}(t)-\theta_{\mathrm{RX}}\left(t-2 t_{p}\right)
\end{aligned}
$$

give phase variations of the transmit and receive laser, respectively, over a time $2 t_{p}=4 \pi \beta_{2} L f_{p}$. Finally, by using the estimator in (13) (or its digital equivalent in (19)) and replacing $\angle y(t)$ with signals $y_{1}(t)$ or $y_{2}(t)$, transmit and receive PN can be separately estimated. In this case, the role played by the DPNE delay $\tau$ is taken by the differential group delay $2 t_{p}$, which can be tuned by properly setting frequencies $\pm f_{p}$.

\section{Simulation Results}

Firstly, we verify by simulation the reliability of the performance parameter defined in (5) and of the analysis of the DCE technique given in Section IV. To this aim, we consider a 100 $\mathrm{Gb} / \mathrm{s}$ transmission on an uncompensated link, characterized by a dispersion of $20000 \mathrm{ps} / \mathrm{nm}$ (i.e., $\sim 1200 \mathrm{~km}$ of standard singlemode fiber, $T_{H} \simeq 4 \mathrm{~ns}$ for DP-QPSK), and ideally remove transmit PN (i.e., we set $\nu_{\mathrm{TX}}=0$ ).

Denoting by $E_{b}$ the received signal energy per information bit and $N_{0}$ the two-sided PSD of each polarization component of the ASE noise low-pass equivalent, Fig. 5 shows the value of $E_{b} / N_{0}$ required to obtain a BER of $10^{-3}$ versus the DPNE delay $\tau$ for an SC or OFDM system and a receive laser linewidth $\nu_{\mathrm{RX}}$ of 5 or $15 \mathrm{MHz}$. The DPNE is implemented with $\alpha P_{\mathrm{LO}}=-12 \mathrm{dBm}$ and $N_{\mathrm{EP}}=10 \mathrm{pW} / \sqrt{\mathrm{Hz}}$. The SC transmitter has an electrical driver with bandwidth $0.8 / T$ and an optical filter with (equivalent low-pass) bandwidth $0.9 / T$. The receive optical filter has bandwidth equal to $0.8 / T$, and an electrical postdetection 5th-order Bessel filter of bandwidth $0.7 / T$ is also present.

In OFDM, the same filters are used, but the receive optical filter has bandwidth $0.5 / T$ since the signal spectrum is narrower 


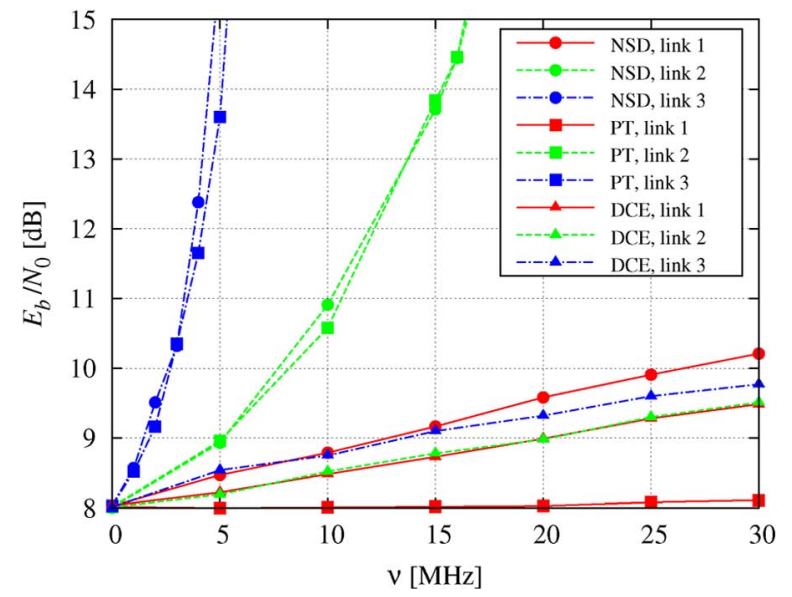

Fig. 6. Required $E_{b} / N_{0}$ vs linewidth $\nu$ to obtain a BER of $10^{-3}$ for $100 \mathrm{~Gb} / \mathrm{s}$ $\mathrm{SC}$ transmission, with or without DCE, on three different links.

and aliasing is to be avoided. All filters are $4^{\text {th }}$-order Gaussian, if not otherwise specified. The simulated OFDM system is characterized by 3232 subcarriers, including 606 virtual subcarriers. The CP has length 256. For the SC system, the length of the two-dimensional adaptive equalizer is 15 taps (whose step size is decreased to $10^{-5}$ after convergence), whereas two fixed-tap equalizers (one per polarization) of length 1600 compensate for the chromatic dispersion. The asynchronous detection strategy is characterized by a memory within 4-8 symbols (optimized case by case, see [4]). These design parameters for SC and OFDM are sufficient, in the absence of phase noise, to avoid penalties with respect to the back-to-back case (a BER of $10^{-3}$ is reached with reference $E_{b} / N_{0}$ of $8 \mathrm{~dB}$ ). Theoretical curves are obtained from (6) and (15), replacing $T_{H}$ with the FFT block length for OFDM. A good agreement between simulations and theory confirms the reliability of the analysis presented in Section IV, both in terms of system penalty and optimum delay. The minimum penalty obtained for an optimum delay $\tau_{\text {opt }}$ is practically negligible (always below $0.3 \mathrm{~dB}$ ) and should be compared with the much higher values in the absence of DCE (1 $\mathrm{dB}$ and more than $5 \mathrm{~dB}$ for $5 \mathrm{MHz}$ and $15 \mathrm{MHz} \mathrm{SC}$, respectively, whereas OFDM does not work). Penalty remains small also for higher-than-optimum delays $\tau$ and, consequently, for narrower-bandwidth DPNE. In a second simulation, we consider a more realistic scenario and compare the performance of SC and OFDM systems on various links deploying different strategies against $\mathrm{PN}$.

We consider three different links: link 1 characterized by 650 $\mathrm{ps} / \mathrm{nm}$ of residual chromatic dispersion (CD), corresponding to approximately $38 \mathrm{~km}$ of standard SMF; link 2 with 20000 ps $/ \mathrm{nm}$ of CD (about $1200 \mathrm{~km}$ ) as in Fig. 5; link 3 with 100000 $\mathrm{ps} / \mathrm{nm}$ of CD (about $5800 \mathrm{~km}$ ). We include also transmit PN and assume that transmit and receive lasers have identical linewidth $\nu$ (i.e., $\nu_{\mathrm{TX}}=\nu_{\mathrm{RX}}=\nu$ ). Fig. 6 shows the performance of an $\mathrm{SC}$ system versus $\nu$ when employing only the asynchronous detection strategy (NSD), an (ideal, see below) PT-based technique (PT), or DCE plus asynchronous detection at the receiver (DCE). A 15-tap two-dimensional adaptive equalizer is used for all links, as the ISI due to CD is always compensated

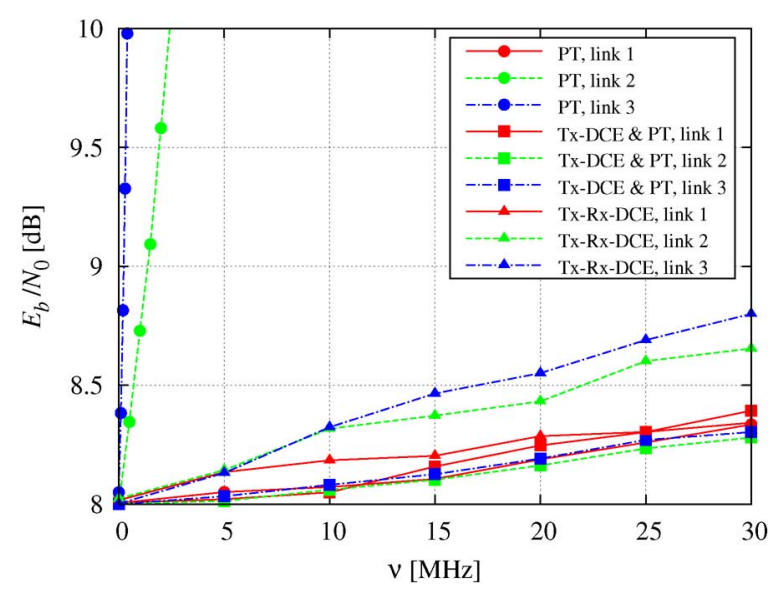

Fig. 7. Required $E_{b} / N_{0}$ vs linewidth $\nu$ to obtain a BER of $10^{-3}$ for $100 \mathrm{~Gb} / \mathrm{s}$ OFDM transmission, with or without DCE, on three different links.

for by two fixed-tap equalizers, one per received polarization, placed before the two-dimensional one. The number of taps of the fixed-tap equalizers is $50,1600,6000$ for link 1, 2, 3, respectively. When the PT-based technique is employed, both for SC and OFDM systems, it is assumed that the phase of the unmodulated carrier is ideally extracted. This is obtained in simulation by transmitting the PT on an identical noise-free parallel (and thus also not affected by the interference due to the information-bearing signal) channel. On link 1, the channel effect is negligible and penalty is small in all considered cases, although lower with DCE, which practically cancels out receive PN, and almost zero with PT which, being ideal, compensates exactly for the sum of transmit and receive PN (though, when the PT is not ideally extracted, a loss must be expected). Despite this ideal estimation, as mentioned in Section III, the PT technique becomes useless in the absence of inline dispersion compensation (link 2 or 3 ), where penalty increases significantly with $\nu$, being similar for the PT compensation or asynchronous detection. On the other hand, DCE exhibits the same (small) penalty on link 1 and 2, mainly due to transmit PN, and only slightly higher on link 3 . Fig. 7 shows the performance of an OFDM system versus $\nu$ when only the ideal PT-based compensation is employed (PT), when PT and transmit-end DCE (Tx-DCE \& PT) are employed, or when transmit- and receive-end DCE (Tx-Rx-DCE) are both employed, without PT. The number of subcarriers, virtual subcarriers and the CP length are, respectively, 128, 24, 16 for link 1; 3232, 606, 256 for link 2; 12928, 2420, 1024 for link 3. In the OFDM case, as expected, the impact of PN is higher than for SC. Even for a short channel memory (link 1), when PN is not affected by channel dispersion, its variations on the FFT block length are not negligible and a PT-based compensation is required to avoid ICI. Similar performance on link 1 is obtained also by employing Tx-DCE \& PT or Tx-Rx-DCE. However, as in the SC case, PT-based compensation fails when PN is affected by channel dispersion (link 2 or 3), while both techniques based on DCE feature a negligible penalty even for large linewidths (the small advantage of Tx-DCE \& PT being due to the deployment of an ideal PT-based compensation) 


\section{CONCLUSION}

The performance of SC and OFDM systems in the presence of transmit and receive phase noise (PN) has been discussed. Different compensation techniques have been considered in both systems with and without inline dispersion compensation, with particular emphasis on the latter. It was shown that SC systems with asynchronous detection and OFDM with pilot tone already provide good performance with available lasers in dispersion-compensated links, whereas they are unable to keep the penalty within an acceptable range in uncompensated systems - the penalty increasing with the bit-rate-distance product and laser linewidth. In particular, it was shown that even an ideal pilot-tone-based compensation is useless in uncompensated SC or OFDM systems because of its inability to distinguish between transmit and receive $\mathrm{PN}$, as required by the presence of channel dispersion. A digital coherence enhancement (DCE) technique was then described that, when employed at transmit (Tx-DCE) or receive ends (Rx-DCE) by using a proper hardware architecture and compensation algorithm, is able to significantly reduce the amount of PN, loosening the constraints on the corresponding laser. Both analogical and digital DCE estimators were derived, and their statistical properties and optimization demonstrated. A simplified DCE implementation and an alternative equivalent technique that, by using a double pilot tone, requires no opto-electronic devices were also discussed. Three different configurations (namely, SC with Rx-DCE and asynchronous detection, OFDM with Tx-DCE and PT, and OFDM with Tx-DCE and Rx-DCE) have been considered and tested by numerical simulations, showing a remarkable PN penalty reduction on dispersion-uncompensated systems with respect to SC or OFDM configurations employing classical PN compensation techniques. As predicted by theory and confirmed by numerical simulations, the effectiveness of DCE increases at higher bit-rate-distance products, whereas its complexity decreases. Therefore, we expect it to be a cost-effective solution for increasing the bit-rate-distance product of next-generation dispersion-uncompensated coherent optical systems with respect to deploying lasers with narrower linewidths.

\section{APPENDIX}

For the sake of simplicity, we consider only one polarization and a QPSK signal, normalized to have unit power, whose pulses are rectangularly shaped in frequency with bandwidth $1 /(2 T)$. After equalization, assuming that the equalizer is ideal in the absence of PN-i.e., that $\sum_{i} c_{i} s_{k-i}=a_{k}$, where $\left\{s_{k}\right\}$ are the samples (at sampling frequency $1 / T$ ) of the received (noiseless) signal affected by GVD, $\left\{c_{i}\right\}$ the $N$ equalizer coefficients, and $\left\{a_{k}\right\}$ the independent transmitted symbols - the received sample can be expressed as

$$
r_{k}=\sum_{i} c_{i} s_{k-i} e^{j \theta_{k-i}}+n_{k}=\left(a_{k}+q_{k}\right) e^{j \theta_{k}}+n_{k}
$$

where $\left\{\theta_{k}\right\}$ is the (residual, if DCE is present) receive PN, $\left\{n_{k}\right\}$ are the normalized independent Gaussian noise samples due to ASE with variance $\sigma_{n}^{2}$, and

$$
q_{k}=\sum_{i} c_{i} s_{k-i}\left[e^{j\left(\theta_{k-i}-\theta_{k}\right)}-1\right]
$$

is the additive noise due to interaction between receive $\mathrm{PN}$ and equalization. It is easy to demonstrate that, despite the presence of ISI induced by GVD, $E\left\{s_{k} s_{j}^{*}\right\}=E\left\{a_{k} a_{j}^{*}\right\}=\delta_{k j}$, where $\delta_{k j}$ is the Kronecker delta, such that $q_{k}$ is the sum of $N$ uncorrelated random variables, each with a random phase. Thus, for large $N, q_{k}$ is well approximated by a zero-mean circularly symmetric Gaussian variable. Since, as shown later, $\theta_{k-i}-\theta_{k}$ is a Gaussian variable with variance $\sigma_{i}^{2}, E\left\{e^{j\left(\theta_{k-i}-\theta_{k}\right)}\right\}=e^{-\sigma_{i}^{2} / 2}$ and

$$
\begin{aligned}
& E\left\{q_{k} q_{k+\ell}^{*}\right\} \\
& \quad=\sum_{i} c_{i} c_{i+\ell}^{*}\left(1-e^{-\sigma_{i}^{2} / 2}-e^{-\sigma_{i+\ell}^{2} / 2}+e^{-\sigma_{\ell}^{2} / 2}\right)
\end{aligned}
$$

An ideal dispersion equalizer is well approximated by $N$ coefficients, spanning over the whole channel memory $T_{H}$, with constant amplitude and quadratically varying phase. Thus

$$
\sigma_{q}^{2}=E\left\{\left|q_{k}\right|^{2}\right\} \simeq 2 \sum_{i}\left(1-e^{-\sigma_{i}^{2} / 2}\right) / N \simeq \overline{\sigma_{\theta}^{2}}
$$

where the last approximation stems from (5) and holds for small $\sigma_{i}^{2}$, while correlation (24) goes rapidly to zero as $|\ell|$ increases. Thus, $r_{k}$ in (22) is affected by the amplifier noise, the slowly varying receive $\mathrm{PN}$, and an additional Gaussian noise, approximately white and with variance (25). Thus, neglecting the slowly varying PN, the BER is given by $Q\left[\left(\sigma_{n}^{2}+\sigma_{q}^{2}\right)^{-1 / 2}\right]$, which easily leads to the analytical approximation (6) for the penalty and to a BER floor of $Q\left(1 / \sigma_{q}\right)$ for vanishingly small amplifier noise, in good agreement with the analysis reported in [32]. A slightly more accurate result could be obtained by accounting also for the small correlation $E\left\{q_{k} a_{k}^{*}\right\}=-\sigma_{q}^{2} / 2$. In the absence of DCE, the received signal is directly affected by receive PN, $\theta_{\mathrm{RX}}(t)$, a Wiener process (i.e., a Gaussian zero-mean process with variance $2 \pi \nu_{\mathrm{RX}} t$ linearly increasing with time) that can be seen as stemming from a white Gaussian frequency noise process $\nu(t)$ with two-sided PSD $\nu_{\mathrm{RX}} / 2 \pi$ through

$$
\theta_{\mathrm{RX}}(t)=2 \pi \int_{-\infty}^{t} \nu(x) d x
$$

In this case, according to the definition in (5), its average variance over channel memory $T_{H}$ is

$$
\overline{\sigma_{\theta}^{2}}=\frac{1}{T_{H}} \int_{-T_{H} / 2}^{T_{H} / 2} 2 \pi \nu_{\mathrm{RX}}|\xi| d \xi=\frac{\pi \nu_{\mathrm{RX}} T_{H}}{2} .
$$

The analysis is slightly more involved in the presence of DCE. Given the LO in (10), the optical signals at the four output ports of the optical hybrid in Fig. 3 are

$$
e_{n}(t)=\sqrt{\frac{\alpha}{8}}\left[e_{\mathrm{LO}}(t)+e_{\mathrm{LO}}(t-\tau) e^{j\left(\phi^{\prime}+n \pi / 2\right)}\right]
$$


where $n=0, \ldots, 3$ and $\phi^{\prime}$ is the unknown phase shift between the delayed and undelayed optical carrier at the input of the optical hybrid. Assuming that the detectors bandwidth is much larger than the laser linewidth $\nu_{\mathrm{RX}}$ and thermal noise dominant over shot noise, the photodetected signals are

$$
\begin{aligned}
x_{r}(t) & =R\left[\left|e_{0}(t)\right|^{2}-\left|e_{2}(t)\right|^{2}\right]+w_{r}(t) \\
x_{i}(t) & =R\left[\left|e_{1}(t)\right|^{2}-\left|e_{3}(t)\right|^{2}\right]+w_{i}(t)
\end{aligned}
$$

where $w_{r}(t)$ and $w_{i}(t)$ are two independent zero-mean white (over the detector bandwidth) Gaussian processes with twosided PSD $2 k_{B} \Theta F_{n} / R_{L}=R^{2} N_{\mathrm{EP}}^{2} / 2$, all the parameters being defined in Section IV. By substituting (10) and (28) in (29) and (30), we finally obtain the normalized complex signal $y(t)$ in (11). When $\tau \ll 1 / \nu_{\mathrm{RX}}$, its PSD is easily evaluated as

$$
P_{y}(f) \simeq \delta(f)+2 \pi \nu_{\mathrm{RX}} \tau^{2} \operatorname{sinc}^{2}(\tau f)+N_{w}
$$

with $N_{w}$ given in (12). Therefore, provided that photodetectors bandwidth is larger than $1 / \tau$, filtering effects on the signal term can be effectively neglected and the noise term $w(t)$ can be considered white over the signal bandwidth. In this case, (11) can be rewritten as

$$
y(t)=\left[1+w^{\prime}(t)\right] e^{j\left[\Delta \theta_{\mathrm{RX}}(t)+\phi\right]}
$$

where $\Delta \theta_{\mathrm{RX}}(t)=\theta_{\mathrm{RX}}(t)-\theta_{\mathrm{RX}}(t-\tau)$ is a r.v., with zero mean and variance $2 \pi \nu_{\mathrm{RX}} \tau$, that equals the variation of receive $\mathrm{PN}$ on a time interval $\tau$, and $w^{\prime}(t)=w_{r}^{\prime}(t)+j w_{i}^{\prime}(t)$ is statistically equivalent to $w(t)$. Assuming a sufficiently high signal-to-noise ratio, such that the following approximation holds

$$
\arctan \left[\frac{w_{i}^{\prime}(t)}{1+w_{r}^{\prime}(t)}\right] \simeq w_{i}^{\prime}(t)
$$

the phase of $y(t)$ can be expressed as

$$
\angle y(t) \simeq \Delta \theta_{\mathrm{RX}}(t)+\phi+w_{i}^{\prime}(t)
$$

with expected value $E\{\angle y(t)\}=\phi$. Thus, an accurate unbiased estimate $\hat{\phi}$ of the constant term $\phi$ can be obtained from a sufficiently-long time average of $\angle y(t)$. By substituting (34) in (13) and assuming that $\hat{\phi}=\phi$, the phase estimate in (13) can be eventually rewritten as

$$
\begin{aligned}
\hat{\theta}(t) & =\frac{1}{\tau} \int_{t_{0}}^{t+\tau / 2}\left[\theta_{\mathrm{RX}}(x)-\theta_{\mathrm{RX}}(x-\tau)+w_{i}^{\prime}(t)\right] d x \\
& =\theta_{\mathrm{RX}}(t)+\theta_{0}+\theta_{w}(t)+\theta_{d}(t)
\end{aligned}
$$

where

$$
\begin{aligned}
\theta_{0} & =\frac{1}{\tau} \int_{t_{0}-\tau}^{t_{0}} \theta_{\mathrm{RX}}(x) d x \\
\theta_{w}(t) & =\frac{1}{\tau} \int_{t_{0}}^{t+\tau / 2} w_{i}^{\prime}(x) d x \\
\theta_{d}(t) & =\frac{1}{\tau} \int_{t-\tau / 2}^{t+\tau / 2} \theta_{\mathrm{RX}}(x) d x-\theta_{\mathrm{RX}}(t)
\end{aligned}
$$

and $w_{i}^{\prime}(t)$ is a stationary Gaussian process with PSD $N_{w} / 2$. Since $\theta_{\mathrm{RX}}(t)$ is the PN to be estimated and removed, while $\theta_{0}$, $\theta_{w}(t)$, and $\theta_{d}(t)$ are, respectively, a constant term and two independent processes, we can evaluate (5) by writing

$$
\begin{aligned}
E\left\{[\theta(t+\xi)-\theta(t)]^{2}\right\}=E\{ & {\left.\left[\theta_{w}(t+\xi)-\theta_{w}(t)\right]^{2}\right\} } \\
& +E\left\{\left[\theta_{d}(t+\xi)-\theta_{d}(t)\right]^{2}\right\} .
\end{aligned}
$$

By comparing (37) with (26), it is apparent that also $\theta_{w}(t)$ is a Wiener process, statistically equivalent to a $\mathrm{PN}$ generated by a laser with linewidth $\nu_{\mathrm{eq}}=N_{w} /\left(4 \pi \tau^{2}\right)$. Therefore, the first term of (39) is

$$
E\left\{\left[\theta_{w}(t+\xi)-\theta_{w}(t)\right]^{2}\right\}=2 \pi \nu_{\mathrm{eq}}|\xi|=\frac{N_{w}|\xi|}{2 \tau^{2}} .
$$

As regards $\theta_{d}(t)$, it can be rewritten as

$$
\theta_{d}(t)=\frac{1}{\tau} \int_{-\tau / 2}^{\tau / 2} \vartheta(t ; x) d x
$$

where $\vartheta(t ; x)=\theta_{\mathrm{RX}}(t+x)-\theta_{\mathrm{RX}}(t)$ is a zero-mean stationary Gaussian process with

$$
E\{\vartheta(t ; x) \vartheta(t ; y)\}= \begin{cases}2 \pi \nu_{\mathrm{RX}} \min \{|x|,|y|\}, & x y>0 \\ 0, & x y \leq 0 .\end{cases}
$$

Therefore, we can evaluate

$$
\begin{aligned}
E\left\{\theta_{d}^{2}(t)\right\} & =\frac{1}{\tau^{2}} \int_{-\tau / 2}^{\tau / 2} \int_{-\tau / 2}^{\tau / 2} E\{\vartheta(t ; x) \vartheta(t ; y)\} d x d y \\
& =\frac{4}{\tau^{2}} \int_{0}^{\tau / 2} \int_{0}^{y} 2 \pi \nu_{\mathrm{RX}} x d x d y=\frac{\pi \nu_{\mathrm{RX}} \tau}{6}
\end{aligned}
$$

and write the second term of (39) as

$$
\begin{aligned}
E\left\{\left[\theta_{d}(t+\xi)-\theta_{d}(t)\right]^{2}\right\} & \\
& =\frac{\pi \nu_{\mathrm{RX}} \tau}{3}-2 E\left\{\theta_{d}(t+\xi) \theta_{d}(t)\right\} .
\end{aligned}
$$

Finally, by averaging the contribution of (40) and (44) for $-T_{H} / 2<\xi<T_{H} / 2$ and neglecting the correlation of $\theta_{d}(t)$ and $\theta_{d}(t+\xi)$ in (44) (that vanishes for $\xi>\tau$ and is therefore negligible, on average, for $\tau \ll T_{H}$ ) the average PN variance defined in (5) is

$$
\overline{\sigma_{\theta \mathrm{DCE}}^{2}} \simeq \frac{\pi \nu_{\mathrm{RX}} \tau}{3}+\frac{N_{w} T_{H}}{8 \tau^{2}} .
$$

\section{REFERENCES}

[1] G. Colavolpe, T. Foggi, E. Forestieri, and M. Secondini, "Phase noise sensitivity and compensation techniques in long-haul coherent optical links," in Proc. IEEE Global Telecommun. Conf., Miami, FL, Dec. 2010.

[2] G. Raybon and P. J. Winzer, " $100 \mathrm{~Gb} / \mathrm{s}$ challenges and solutions," in Proc. Optical Fiber Commun. Conf. (OFC'08), San Diego, CA, Feb. 2008, Paper OTuG1.

[3] L. G. Kazovsky, "Homodyne phase-shift-keying systems: Past challenges and future opportunities," J. Lightw. Technol., vol. 24, pp. 4876-4884, Dec. 2006. 
[4] G. Colavolpe, T. Foggi, E. Forestieri, and G. Prati, "Robust multilevel coherent optical systems with linear processing at the receiver," J. Lightw. Technol., vol. 27, no. 13, pp. 2357-2369, July 1, 2009.

[5] J. A. C. Bingham, "Multicarrier modulation for data transmission: An idea whose time has come," IEEE Commun. Mag., pp. 5-14, May 1990

[6] S. L. Jansen, B. Spinnler, I. Morita, S. Randel, and H. Tanaka, "100 GbE: QPSK versus OFDM,” Opt. Fiber Technol., vol. 15, no. 5-6, pp. 407-413, Oct.-Dec. 2009.

[7] W. Shieh, X. Yi, and Q. Yang, "Coherent optical OFDM: Has its time come?," J. Opt. Netw., vol. 7, no. 3, pp. 234-255, Mar. 2008.

[8] J. Armstrong, "OFDM for optical communications," J. Lightw. Technol., vol. 27, no. 3, pp. 189-204, Feb. 2009.

[9] A. Barbieri, G. Colavolpe, T. Foggi, E. Forestieri, and G. Prati, "OFDM vs. single-carrier transmission for 100 Gbps optical communication," J. Lightw. Technol., vol. 28, no. 17, pp. 2537-2551, Sep. 1, 2010.

[10] E. Ip and J. M. Kahn, "Carrier synchronization for 3- and 4-bit-persymbol optical transmission," J. Lightw. Technol., vol. 23, no. 12, pp. 4110-4124, Dec. 2005.

[11] T. Pfau, S. Hoffmann, and R. No, "Hardware-efficient coherent digital receiver concept with feedforward carrier recovery for M-QAM constellations," J. Lightw. Technol., vol. 27, no. 8, pp. 989-999, Apr. 2009.

[12] U. Mengali and A. N. D'Andrea, Synchronization Techniques for Digital Receivers (Applications of Communications Theory). New York: Plenum Press, 1997.

[13] R. Noè, "Phase noise-tolerant synchronous QPSK/BPSK basebandtype intradyne receiver concept with feedforward carrier recovery," $J$ Lightw. Technol., vol. 23, pp. 802-808, Feb. 2005.

[14] D.-S. Ly Gagnon, S. Tsukamoto, K. Katoh, and K. Kikuchi, "Oherent detection of optical quadrature-phase-shift-keying signal with carrier phase estimation," J. Lightw. Technol., vol. 24, pp. 12-21, Jan. 2006.

[15] E. Ip and J. M. Kahn, "Feedforward carrier recovery for coherent optical communications," J. Lightw. Technol., vol. 25, pp. 2675-2692, Sep. 2007.

[16] G. Colavolpe and R. Raheli, "Noncoherent sequence detection," IEEE Trans. Commun., vol. 47, pp. 1376-1385, Sep. 1999.

[17] G. Colavolpe, A. Barbieri, and G. Caire, "Algorithms for iterative decoding in the presence of strong phase noise," IEEE J. Sel. Areas Commun., vol. 23, no. 9, pp. 1748-1757, Sep. 2005.

[18] G. Colavolpe, "On LDPC codes over channels with memory," IEEE Trans. Wireless Commun., vol. 5, no. 7, pp. 1757-1766, July 2006.

[19] G. Charlet, S. Bigo, J. Renaudier, O. B. Pardo, M. Salsi, H. Mardoyan, and P. Tran, "The impact and mitigation of non-linear effects in coherent optical transmission," in Proc. Opt. Fiber Commun. Conf. (OFC'09), San Diego, CA, Mar. 2009, Paper NThB4.

[20] E. M. Ip and J. M. Kahn, "Fiber impairment compensation using coherent detection and digital signal processing," J. Lightw. Technol., vol. 28, no. 4, pp. 502-519, Feb. 2010.

[21] L. G. Kazowsky, S. Benedetto, and A. Willner, Optical Fiber Communication Systems. Norwood, MA: Artec House, 1996.

[22] L. Tomba, "On the effect of Wiener phase noise in OFDM systems," IEEE Trans. Commun., vol. 46, no. 5, pp. 580-583, May 1998.

[23] S. L. Jansen, I. Morita, T. C. W. Schenk, N. Takeda, and H. Tanaka, "Coherent optical 25.8-Gb/s OFDM transmission over 4160-km SSMF," J. Lightw. Technol., vol. 26, no. 1, pp. 6-15, Jan. 2008.

[24] S. Benedetto, E. Biglieri, and V. Castellani, Digital Transmission Theory. Englewood Cliffs, NJ: Prentice-Hall, 1987.

[25] E. Forestieri and G. Prati, "Novel optical line codes tolerant to fiber chromatic dispersion," J. Lightw. Technol., vol. 19, no. 11, pp. 1675-1684, Nov. 2001.

[26] W. Shieh and K. P. Ho, "Equalization-enhanced phase noise for coherent detection systems using electronic digital signal processing," Opt. Exp., vol. 16, pp. $15718-15$ 727, Sept. 2008.

[27] A. P. T. Lau, T. S. R. Shen, W. Shieh, and K.-P. Ho, "Equalization-enhanced phase noise for $100 \mathrm{~Gb} / \mathrm{s}$ transmission and beyond with coherent detection," Opt. Exp., vol. 18, no. 16, pp. 17 239-17 251, Aug. 2010.

[28] G. P. Agrawal, Fiber-optic Communications Systems, 3rd ed. New York: Wiley, 2002.

[29] M. Secondini, G. Meloni, T. Foggi, G. Colavolpe, L. Poti, and E. Forestieri, "Phase noise cancellation in coherent optical receivers by digital coherence enhancement," in Proc. Eur. Conf. Opt. Commun (ECOC'10), Torino, Italy, Sep. 19-23, 2010.

[30] P. Kabal and S. Pasupathy, "Partial-response signaling," IEEE Trans. Commun., vol. 23, no. 9, pp. 921-934, Sep. 1975.

[31] N. McGinty, R. Kennedy, and P. Hocher, "Parallel trellis viterbi algorithm for sparse channels," IEEE Commun. Lett., vol. 2, no. 5, pp. 143-145, May 1998.
[32] T. Xu, G. Jacobsen, S. Popov, J. Li, A. T. Firber, and Y. Zhang, “Analytical estimation of phase noise influence in coherent transmission system with digital dispersion equalization," Opt. Exp., vol. 19, no. 8, pp. 7756-7768, Apr. 2011.

Giulio Colavolpe (SM'09) was born in Cosenza, Italy, in 1969. He received the Dr. Ing. degree in telecommunications engineering (cum laude) from the University of Pisa, Italy, in 1994 and the Ph.D. degree in information technologies from the University of Parma, Italy, in 1998.

Since 1997, he has been at the University of Parma, Italy, where he is now an Associate Professor of Telecommunications. In 2000, he was Visiting Scientis at the Institut Eurécom, Valbonne, France. His main research interests include digital transmission theory, adaptive signal processing, channel coding and information theory. His research activity has led to several scientific publications in leading international journals and conference proceedings and a few industrial patents. He is also co-author of the book Detection Algorithms for Wireless Communications, with Applications to Wired and Storage Systems (Wiley, 2004).

Dr. Colavolpe is also the principal investigator of several research projects funded by the European Space Agency (ESA-ESTEC) and important telecommunications companies. In 2005, he received the best paper award at the 13th International Conference on Software, Telecommunications and Computer Networks (SoftCOM'05).

Tommaso Foggi was born in Parma, Italy, on May 7, 1978. He received the $\mathrm{Dr}$. Ing. degree in telecommunications engineering and the Ph.D. degree in information technology from the University of Parma, Italy, in 2003 and 2008, respectively.

From July 2003 to July 2004 he was granted a CNIT scholarship at Photonic Networks National Laboratory, Pisa, and another CNIT scholarship from July 2005 to July 2006 at Dipartimento di Ingegneria dell'Informazione (DII), Università Degli Studi di Parma, Parma. Since June 2009 he is now a research fellow of National Inter-university Consortium for Telecommunications (CNIT), after holding post-doc collaboration contracts with University of Parma, CNIT and Scuola Superiore Sant'Anna in 2008 and 2009. His main research interests include digital signal processing for optical communications, i.e., equalization, estimation and compensation techniques, advanced modulation formats, detection algorithms and receiver design. His activity also contributed to several research projects funded by the Italian Ministry of Education, University and Research (MIUR) or by International companies.

Enrico Forestieri (S'91-M'92) received the Dr. Ing. degree in electronics engineering from the University of Pisa, Pisa, Italy, in 1988.

From 1989 to 1991 he has been a postdoctoral scholar at the University of Parma, Italy, working on optical communication systems. From 1991 to 2000 he was a Research Scientist and faculty member of the University of Parma. Since 2001 he is with Scuola Superiore Sant'Anna di Studi Universitari e di Perfezionamento, Pisa, Italy, where currently he is Professor of Telecommunications. His research interests are in the general area of digital communication theory and optical communication systems, with special attention to adaptive optical and electronic equalization, channel coding, and advanced modulation formats for optical systems. He is the leader of the "Optical Transmission Theory and Techniques" area at the Integrated Research Center for Photonic Networks and Technologies (IRCPhoNeT), Pisa, Italy. His research activity has led to numerous scientific publications in leading international journals and conference proceedings, as well as a few patents. In 2004 he was General Chairman of the Tyrrhenian International Workshop on Digital Communications.

Marco Secondini received the Dr. Ing. degree in electronics engineering from the University of Roma Tre, Rome, in 2000, and the Ph.D. degree from Scuola Superiore Sant'Anna, Pisa, in 2006.

During 2001, he was with QPlus Networks Inc., developing fiber optic systems for ultra long-haul communications. Since 2002, he has been with Scuola Superiore Sant'Anna, where he is currently an Assistant Professor. During 2005, he was a Visiting Faculty Research Assistant in the Photonics Group, University of Maryland Baltimore County, Baltimore. His current research interests are in the area of optical communication theory and include: polarization mode dispersion, fiber nonlinearities, coherent optical systems, equalization techniques. 